\title{
Measurements of W/Z boson production in association with jets at ATLAS
}

\author{
Camilla Vittori, on behalf of the ATLAS Collaboration ${ }^{a, *}$ \\ a INFN and University of Bologna, \\ via Irnerio 46, Bologna, Italy \\ E-mail: camilla.vittori@cern.ch
}

\begin{abstract}
Measurements of W/Z-boson production in association with jets provide important test of perturbative QCD prediction and also yield information about the parton distribution functions of the proton. In this talk, differential cross-sections for vector-boson production in association with jets using proton-proton collisions collected by the ATLAS experiment at the Large Hadron Collider (LHC) are presented. The data are corrected for detector inefficiency and resolution and compared to state-of-the-art theoretical predictions. The impact of the choice of parton distribution function is also presented.
\end{abstract}

40th International Conference on High Energy physics - ICHEP2020

July 28 - August 6, 2020

Prague, Czech Republic (virtual meeting)

\footnotetext{
${ }^{*}$ Speaker
} 


\section{Introduction}

The measurement of the $\mathrm{Z}$ boson production in association with jets in proton-proton collisions provides an important test of perturbative $\mathrm{QCD}$. Theoretical predictions for $\mathrm{Z}+$ jets production are available at NNLO accuracy. In the particular case of $b$-jets, predictions for $\mathrm{Z}+\mathrm{b}$-jets processes can be derived in either the 4-flavour number scheme (4FNS) or the 5-flavour number scheme (5FNS). In the 4FNS, b-quarks to not contribute to the proton parton distribution functions (PDFs) and appear in the final state due to gluon splitting $(\mathrm{g} \rightarrow \mathrm{bb})$. In the 5FNS, the b-quark is considered massless and the b-quark density is allowed in the initial state; therefore in the 5FNS the Z+b-jets cross section is sensitive to the b-quark PDF in the proton.

Measurements of $\mathrm{Z}+(\mathrm{b})$ jets processes provide a benchmark for fixed-order calculations and predictions for Monte Carlo (MC) simulations, which are used to estimate the $Z+(b) j e t s$ background in other topologies, such as the Higgs boson production or in searches for new physics.

In this paper, two measurements performed by the ATLAS experiment of LHC [1] on proton-proton collision data are presented: the production cross sections for $\mathrm{Z}+$ jets at $\sqrt{s}=8 \mathrm{TeV}$ and $\mathrm{Z}+\mathrm{b}$-jets at $\sqrt{s}=13 \mathrm{TeV}$.

\section{Measurement of the inclusive $\mathrm{Z}+$ jets cross-section at $\sqrt{s}=8 \mathrm{TeV}$}

The inclusive jet production in association with a $\mathrm{Z}$ boson is studied in processes where the $\mathrm{Z}$ boson decays into an electron-positron pair. The double differential cross section is measured as a function of the jet absolute rapidity $\left(\left|y_{\text {jet }}\right|\right)$ and transverse momentum $\left(p_{\mathrm{T}}^{\mathrm{jet}}\right)$ using the data collected by the ATLAS detector at $\sqrt{s}=8 \mathrm{TeV}$, corresponding to a luminosity of $19.9 \mathrm{fb}^{-1}$.

Events are selected if they contain a pair of opposite-charge leptons belonging to the decay of the $\mathrm{Z}$ boson and at least one jet. Electrons are required to have transverse momentum $p_{\mathrm{T}}>20 \mathrm{GeV}$, pseudorapidity $|\eta|<2.47$ (excluding the detector transition region) and invariant mass $66 \mathrm{GeV}<m_{\mathrm{ee}}<116 \mathrm{GeV}$. Jets are selected with $p_{\mathrm{T}}>25 \mathrm{GeV}$ and $|y|<3.4$. The topology of the $\mathrm{Z}+$ jets events is easy to identify and clean from background processes, offering a probe for QCD studies. The irreducible background is composed by $t \bar{t}$, which is the dominant contribution, $Z \rightarrow \tau \tau$, diboson and single top-quark processes, modelled by MC samples. Background from multijet and $\mathrm{W}+$ jets events is extracted as a combined template by using a data-driven technique.

The $\mathrm{Z}+\mathrm{jets}$ data events passing the detector level selection are corrected for detector resolution and efficiency to the particle level through an iterative Bayesian unfolding, in order to compare the results with theoretical predictions. The measured double differential cross section covers five orders of magnitude and falls steeply as a function of $\left|y_{\text {jet }}\right|$ and $p_{\mathrm{T}}^{\text {jet }}$. The unfolded data are compared with several MC predictions and fixed-order calculations, as presented in Figure 1. The ratio of the NLO SHERPA 1.4 to data is flat as a function of $\left|y_{\text {jet }}\right|$, but shows a dependence on $p_{\mathrm{T}}^{\text {jet }}$ : the $10 \%$ disagreement in $25 \mathrm{GeV}<p_{\mathrm{T}}^{\text {jet }}<50 \mathrm{GeV}$ improves to few percent for $p_{\mathrm{T}}^{\text {jet }}>200 \mathrm{GeV}$. The LO ALPGEN+PY6 predictions agree with data in the $25 \mathrm{GeV}<p_{\mathrm{T}}^{\text {jet }}<100 \mathrm{GeV}$ region, but exceed data by $20 \%$ at high $p_{\mathrm{T}}^{\mathrm{jet}}$. The NLO SHERPA 2.2 shows a good agreement with data in all the considered bins. The fixed-order theoretical predictions are corrected for the non-perturbative and QED emission effects. The NLO MCFM predictions are 5-10\% lower than data, within the uncertainties. The NNLOJET calculations show a better agreement with data in all of the measured bins. The 



Figure 1: The double-differential Z+jets cross-section as a function of $\left|y_{\text {jet }}\right|$ in the $25 \mathrm{GeV}<p_{\mathrm{T}}^{\text {jet }}<50 \mathrm{GeV}$ (left) and $400 \mathrm{GeV}<p_{\mathrm{T}}^{\text {jet }}<1050 \mathrm{GeV}$ (right) ranges [2].

uncertainties on data are smaller than the uncertainties on the predictions on most of the bins. The measured Z+jets production cross-sections are compared with fixed-order predictions calculated with different PDF sets. In general, the alternative PDFs agree within 1-2\% with the nominal CT14 PDF and the differences are covered by the theoretical uncertainties [2]. A quantitative comparison between the fixed-order NNLOJET, corrected for electroweak and non-perturbative effects, and the measured cross section is performed using a $\chi^{2}$ minimisation and including the experimental and theoretical uncertainties as nuisance parameters. A good agreement is found for $p_{\mathrm{T}}^{\text {jet }}>50 \mathrm{GeV}$ and it is coherent among different PDF sets. Therefore the double differential Z+jets cross section measurement provides a precision input to constrain PDFs.

\section{Measurement of the $\mathrm{Z}+\mathrm{b}$-jets cross-section at $\sqrt{s}=13 \mathrm{TeV}$}

Inclusive and differential cross sections of the $\mathrm{Z}$ boson production in association with at least one or at least two b-jets are measured, considering the electron and muon decay channels of the $\mathrm{Z}$ boson. Data are collected by the ATLAS detector at $\sqrt{s}=13 \mathrm{TeV}$ and correspond to a luminosity of $35.6 \mathrm{fb}^{-1}$.

The associated production of a $\mathrm{Z}$ boson with $\mathrm{b}$-jets is more rare than with inclusive jets $(\mathrm{Z}+\mathrm{jets})$ and $\mathrm{b}$-jets are more challenging to measure. The $\mathrm{b}$-jets are identified by using a multivariate algorithm (MV2c10), which exploits the long lifetime of b-hadrons produced in the quark hadronisation. The b-jets are selected with an efficiency of $70 \%$ of the b-tagging algorithm and with $p_{\mathrm{T}}^{\mathrm{jet}}>20 \mathrm{GeV}$ and $\left|y_{\text {jet }}\right|<2.5$. Electrons and muons are required to be isolated and with $p_{\mathrm{T}}>27 \mathrm{GeV}$ and $|\eta|<2.5$ for muons and 2.47 for electrons. Events are selected if they contain a pair of opposite charge electrons or muons with invariant mass in the $76 \mathrm{GeV}<m_{11}<106 \mathrm{GeV}$ range and if containing at least one b-jet [3]. An additional cut on the missing transverse energy $\left(E_{\mathrm{T}}^{\mathrm{miss}}\right)$ is added in order to reduce the contamination from $t \bar{t}$ events: $E_{\mathrm{T}}^{\text {miss }}<60 \mathrm{GeV}$ in events with $p_{\mathrm{T}}^{\mathrm{Z}}<150 \mathrm{GeV}$. 



Figure 2: (left) Distribution of events as a function of the invariant mass for combined lepton channels. (right) Relative systematic uncertainties in the cross section as a function of $\mathrm{Z} p_{\mathrm{T}}[3]$.

Background coming from $t \bar{t}$, single-top, diboson, $Z \rightarrow \tau \tau$, $\mathrm{W}+\mathrm{jets}$ and $\mathrm{VH}(\rightarrow \mathrm{bb})$ processes are simulated with Monte Carlo generators. The $t \bar{t}$ represents the dominant background in the $\mathrm{Z}+\geq 2$ b-jets $(23 \%)$ and it is validated in an enriched region, with a pair of opposite-flavour leptons $(e \mu)$, and compared with modelling systematic uncertainties.

The $\mathrm{Z}+$ light/c-jets background dominates in the $\mathrm{Z}+\geq 1$ bjet region (36\%). Its normalisation is estimated with a maximum likelihood fit to data of MV2c10 distributed in b-jets calibration bins. The prefit distributions of $Z+l i g h t / c-j e t s$ are scaled for the normalisation factors obtained from the fit. The $\mathrm{Z}+$ light/cjet background is validated in a dedicated region, defined by events containing at least one $b$-jet passing in the 77-70\% range of b-tagging efficiency. A perfect Data/Monte Carlo agreement is found within the flavour tagging uncertainties.

The multijet background is studied with a template fit on data in enriched control regions. The contribution is found to be $<1 \%$ and therefore negligible.

Figure 2(left) shows the agreement between data and predictions for the invariant mass distribution in $\mathrm{Z}+\geq 1$ bjet events. The electron and muon channels are combined to increase the statistics and the precision, after verifying the compatibility of the cross sections of the single lepton channel. Distributions of background subtracted data are corrected for detector level effects to the fiducial phase space at particle level [3] by using an iterative Bayesian unfolding.

Figure 2(right) shows the different contributions to the total systematic uncertainty on the measurement for the $\mathrm{Z}$ boson $p_{\mathrm{T}}$ observable. The dominant detector level uncertainty is the modelling of the b-tagging efficiency, with an impact of $7 \%$ for $Z+\geq 1$ bjet and $14 \%$ for $Z+\geq 2$ b-jets in inclusive cross sections. In the $\mathrm{Z}+\geq 1$ bjet region, the dominant uncertainty on background is due to $\mathrm{Z}+$ light/c-jets processes $(4.5 \%)$ and the impact on the differential cross section affects mostly extreme corners of the phase space (up to $25 \%$ ), as visible in Figure 2(right). In the $Z+\geq 2$ b-jets region, the modelling of $t \bar{t}$ provides the largest uncertainty among all the background with a contribution of $3.8 \%$ to the inclusive cross section and ranges from $1 \%$ to $9 \%$ in the differential measurements. Another contribution to the total uncertainty is given by the unfolding method ( $4 \%$ in the inclusive cross 

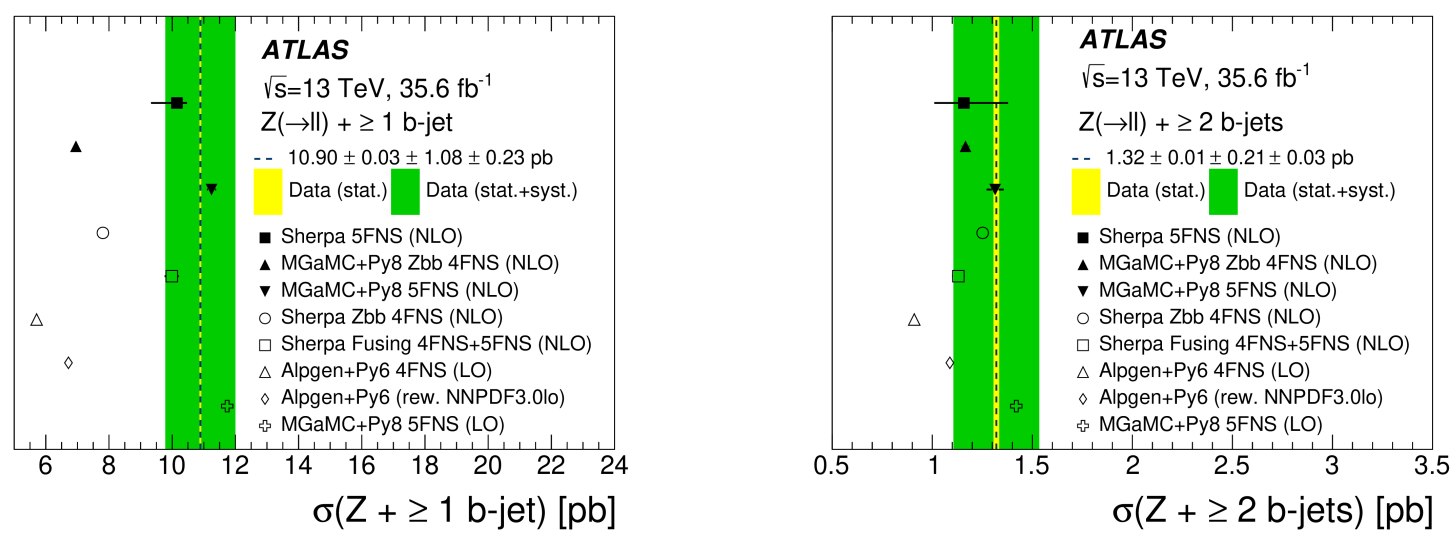

Figure 3: Inclusive cross section for $\mathrm{Z}+\geq 1$ bjet (left) and $\mathrm{Z}+\geq 2 \mathrm{~b}$-jets (right) processes [3].

section).

Data are compared with several predictions with matrix-element at LO or NLO accuracy in both 4FNS or 5FNS. The measurements of the inclusive $\mathrm{Z}+\geq 1$ bjet and $\mathrm{Z}+\geq 2$ b-jets cross sections are presented in Figure 3. The 4FNS predictions are lower than data in the $Z+\geq 1$ bjet region for both LO and NLO matrix-element MC generators, while they agree well with data in the $Z+\geq 2$ b-jets region. The 5FNS predictions provide a better description of the measured cross sections, which is consistent with the result at $\sqrt{s}=7 \mathrm{TeV}$ presented by ATLAS [4].

The differential cross section for $Z+\geq 1$ bjet processes is measured as a function of several observables related to the $\mathrm{Z}$ boson and the leading bjet. In general, the best description of data in provided by Sherpa 5FNS (NLO). The same level of agreement is confirmed by Sherpa Fusing 4FNS+5FNS (NLO), with the exception of the leading bjet $p_{\mathrm{T}}$, where it underestimates the data at high values (see Figure 4 (left)). The high $p_{\mathrm{T}}$ region is better described by MGaMC+Pythia8 5FNS (LO) rather than MGaMC+Pythia8 5FNS (NLO), due to the larger number of partons present in the matrix-element. ALPGEN+Pythia8 predictions well describe the shape of data, despite the large underestimation of the normalisation.

The differential cross section for $Z+\geq 2$ b-jets processes is measured as a function of many different observables related to $b$-jets pairs and $Z$ boson. In general, the best agreement with the measured cross sections is provided by Sherpa 5FNS and Sherpa Fusing 4FNS+5FNS (NLO). In Figure 4 (right) the differential cross section as a function of the angular separation of the two b-jets $\left(\Delta R_{\mathrm{bb}}\right)$ is presented. All Sherpa predictions well describe the shape of data, with a substantial improvement at low $\Delta R_{\mathrm{bb}}$, dominated by the gluon splitting production of the $\mathrm{Zbb}$ final state, relative to the LO version used in the measurement performed by ATLAS at $\sqrt{s}=7 \mathrm{TeV}$ [4]. A significant discrepancy, common to all generators, is found for large values of $m_{\mathrm{bb}}$. The pure Zbb simulation at NLO in the 4FNS, shows significant deviations from the data.

\section{Conclusions}

In this paper, the most recent ATLAS measurements of $Z$ boson production in association with jets are presented: the inclusive $Z+j$ ets cross section at $\sqrt{s}=8 \mathrm{TeV}$ and the inclusive and differential 

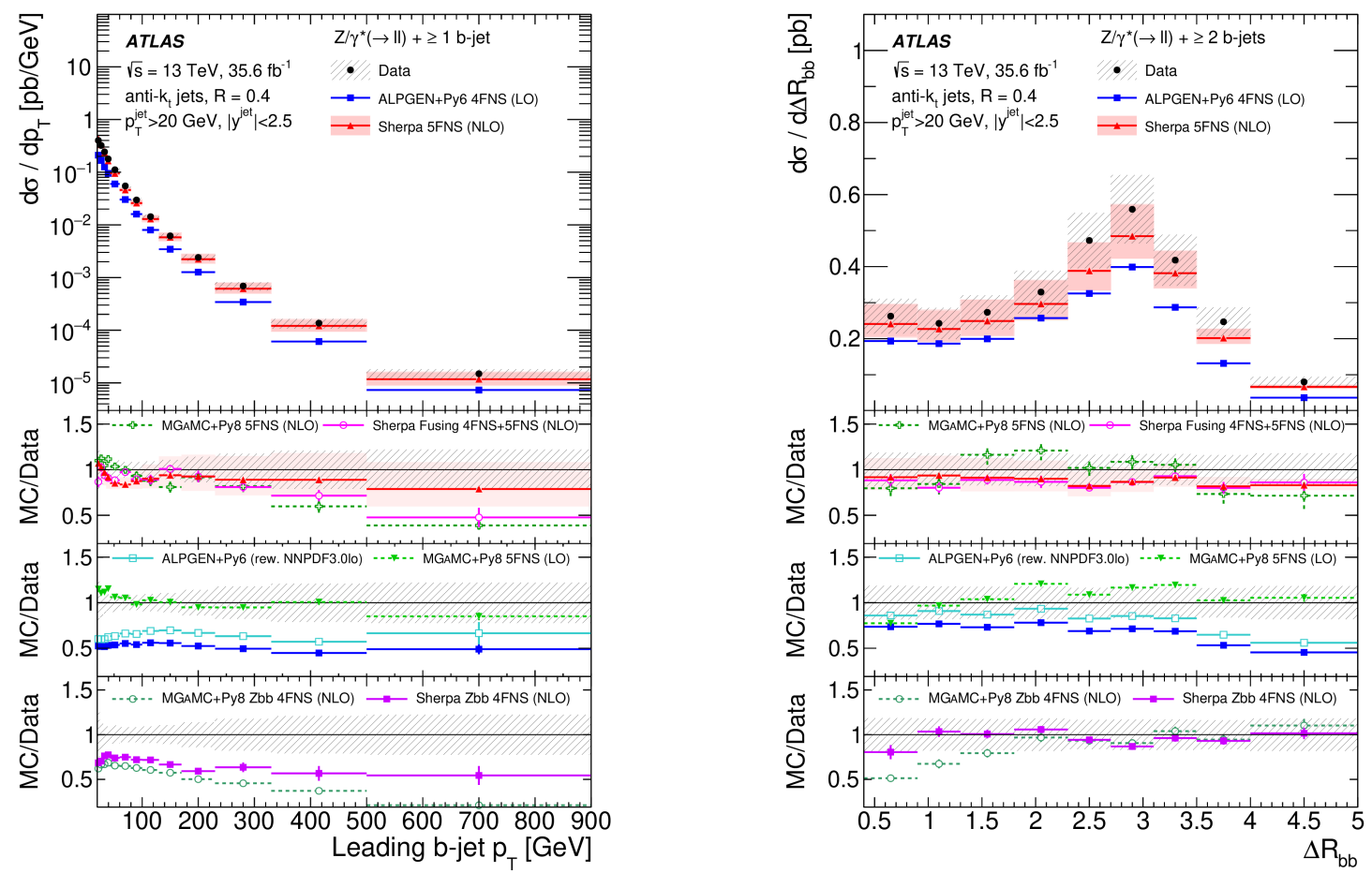

Figure 4: Differential cross section as a function of leading bjet $p_{\mathrm{T}}$ (left) and of the $\Delta R_{\mathrm{bb}}$ (right) for events where the $\mathrm{Z}$ boson is produced in association with at least one or at least $2 \mathrm{~b}$-jets respectively [3].

cross sections for $\mathrm{Z}+\geq 1$ bjet and $\mathrm{Z}+\geq 2$ b-jets processes at $\sqrt{s}=13 \mathrm{TeV}$, performed with data from proton-proton collisions at LHC. In both analyses the results are compared with several theoretical predictions. In general, the measurements provide essential inputs for the improvements of the theoretical predictions, allowing a better quantitative understanding of perturbative QCD and for global fit PDFs.

\section{References}

[1] ATLAS Collaboration, The ATLAS Experiment at the CERN Large Hadron Collider, JINST 3 S0800.

[2] ATLAS Collaboration, Measurement of the inclusive cross-section for the production of jets in association with a $\mathrm{Z}$ boson in proton-proton collisions at $8 \mathrm{TeV}$ using the ATLAS detector, Eur. Phys. J. C 79 (2019) 847.

[3] ATLAS Collaboration, Measurements of the production cross-section for a $Z$ boson in association with $b$-jets in proton-proton collisions at $\sqrt{s}=13 \mathrm{TeV}$ with the ATLAS detector, JHEP 07 (2020) 044.

[4] ATLAS Collaboration, Measurement of differential production cross-sections for a $Z$ boson in association with b-jets in $7 \mathrm{TeV}$ proton-proton collisions with the ATLAS detector, JHEP 10 (2014) 141. 